\title{
TIME DOMAIN COMPUTATION OF A NONLINEAR NONLOCAL COCHLEAR MODEL WITH APPLICATIONS TO MULTITONE INTERACTION IN HEARING*
}

\author{
JACK $\mathrm{XIN}^{\dagger}$, YINGYONG QI ${ }^{\ddagger}$, AND LI DENG ${ }^{\S}$
}

\begin{abstract}
A nonlinear, nonlocal cochlear model of the transmission line type is studied in order to capture the multitone interactions and resulting tonal suppression effects. The model can serve as a module for voice signal processing, and is a one-dimensional (in space) damped dispersive nonlinear PDE based on the mechanics and phenomenology of hearing. It describes the motion of the basilar membrane (BM) in the cochlea driven by input pressure waves. Both elastic damping and selective longitudinal fluid damping are present. The former is nonlinear and nonlocal in BM displacement, and plays a key role in capturing tonal interactions. The latter is active only near the exit boundary (helicotrema), and is built in to damp out the remaining long waves. The initial boundary value problem is numerically solved with a semi-implicit second order finite difference method. Solutions reach a multi-frequency quasi-steady state. Numerical results are shown on two tone suppression from both high-frequency and low-frequency sides, consistent with known behavior of two tone suppression. Suppression effects among three tones are demonstrated by showing how the response magnitudes of the fixed two tones are reduced as we vary the third tone in frequency and amplitude. We observe qualitative agreement of our model solutions with existing cat auditory neural data. The model is thus a simple and efficient processing tool for voice signals.
\end{abstract}

\section{Introduction}

Voice signal processing algorithms have received increasing attention in recent years for improving the design of hearing devices and for evaluating acoustic theories of peripheral auditory systems (see Meddis et al. [22] among others). A fundamental issue a computational method needs to resolve is the nonlinear interaction of acoustic waves of different frequencies and the resulting tonal suppression effects. A nonlinear filter bank approach is developed in [22], based on knowledge of auditory responses. Nonlinearities are known to originate in the cochlea and are further modified in higher level auditory pathways. The cochlear mechanics have first principle descriptions, and so partial differential equations (PDEs) form a natural mathematical framework to initiate computation. However, in vivo cochlear dynamics is not a pure mechanical problem, and neural couplings are present to modify responses. To incorporate both of these aspects, we aim to develop a first principle-based PDE approach to voice signal processing, where the neural aspect is introduced in the model phenomenologically.

Cochlear modeling has had a long history, and various linear models have been studied at length by analytical and numerical methods (see Keller and Neu [14], Leveque, Peskin and Lax [16], Lighthill [18], and references therein). It has been realized that nonlinearity is essential for multitone interactions (see $[11,15,2,8]$ among others). The nonlinearity could be incorporated through micro-mechanics of cochlea, such as coupling of basilar membrane (BM) to inner hair cells [13, 19]. Alternatively, nonlinearity could be introduced phenomenologically based on spreading of electrical and neural activities between hair cells at different BM locations as suggested by experimental data (see Jau and Geisler [12] and Deng [3]). The latter treatment turned

*Received: June 2, 2002; accepted (in revised version): September 18, 2002.

${ }^{\dagger}$ Department of Mathematics and TICAM, University of Texas at Austin, Austin, TX 78712, USA. Corresponding author, (jxin@math.utexas.edu). This work was partially supported by ARO grant DAAD 19-00-1-0524.

${ }^{\ddagger}$ Qualcomm Inc, 5775 Morehouse Drive, San Diego, CA 92121, USA.

$\S$ Microsoft Research, One Microsoft Way, Redmond, WA 98052, USA. 
out to be quite efficient and will be adopted in this paper. Their model with nonlinear and nonlocal BM damping will be our starting point.

Multitone interaction requires one to perform numerical simulation and analysis in the time domain as the resulting time dependent problem is strictly speaking irreducible to a steady state problem. The commonly used cochlear models, including those of [12] and [3], are intrinsically dispersive. In particular, long waves tend to propagate with little decay from the entrance point (stapes) to the exit point (helicotrama). Such an issue can be avoided in linear models because a reduction to the steady state (or a frequency domain calculation using time harmonic solutions) is available by factoring out the time dependence of solutions. The dispersive phenomenon prompted us to incorporate a selective fluid longitudinal damping term in the model of Jau and Geisler [12] and Deng [3], operating near the exit point. The role of such a term is to suck out the long waves accumulating near the exit. Selective positive or negative damping has been a novel way to filter images in PDE method of image processing (see Osher and Rudin [24] for the first work in this direction). Our treatment here is similar in spirit, though on a different type of PDE.

We then present a second order semi-implicit finite difference method of our model. The method relaxes the stability time step constraint of explicit methods without going into the complexity of fully implicit schemes. As our model is nonlocal and nonlinear, a semi-implicit scheme is a reasonable option for computation. Numerical examples showed that the method is efficient, and that the selective damping term indeed removes the long waves at the exit boundary point for a time domain calculation of a single tone. The examples also demonstrated that the analysis of dispersive waves and slow decay of long waves is robust, and the phenomenon persists in the nonlinear nonlocal regime of the model.

Subsequently, we show computational results on isodisplacement curves for five characteristic frequencies consistent with those of Neely [23] for BM displacement of $1 \mathrm{~nm}$. In the case of two tone interactions, our model gives both low and high side suppression, in qualitative agreement with similar studies of Geisler [8]. For three tone interactions, we fix the frequencies and amplitudes of two tones, and vary the third tone both in frequency and amplitude in a manner similar to the auditory neural experiment of Deng and Geisler [4]. Again, our model showed a suppressing effect on the two tones by the third tone, which is in agreement with experimental data. To our best knowledge, the current paper is the first on time domain simulation of interactions of three tones using first principle-based PDEs. The stage is set for further computation, analysis, and model development on more complicated tones.

The rest of the paper is organized as follows. In section 2, we present our model and its background, and analysis of dispersive properties of solutions. In section 3, we outline our semi-implicit discretization of the model. In section 4, we give model parameters, and show numerical results on dispersion of model solutions, isodisplacement curves, two and three tone interactions in comparison with existing data. The conclusions are in section 5 .

\section{Nonlinear Nonlocal Cochlear Model}

\subsection{Background and Model Setup}

The cochlear modeling has had a long history driven by advancement of cochlear experiments (see $[28,25,1,8]$ among others).

A brief derivation of the one dimensional (1-D) cochlear model of transmission line (long wave) type, based on fluid mechanics and elasticity, can be found in Sondhi [26] (see also references therein for earlier contributions, and de Boer [2] for later 
development and higher dimensional models). A general form of the 1-D model can be expressed as:

$$
\begin{aligned}
& p_{x x}-N u_{t t}=\epsilon_{s} u_{t}, \quad x \in(0, L), \\
& p=m u_{t t}+r(x, u) u_{t}+s(x) u,
\end{aligned}
$$

where $p$ is the fluid pressure difference across the basilar membrane (BM), $u$ the $\mathrm{BM}$ displacement, $L$ the longitudinal length of BM; stapes is at $x=0$, and helicotrema is at $x=L ; N$ is a constant depending on fluid density and cochlear channel size; $\epsilon_{s}$ depends on the damping of longitudinal fluid motion [20]; $m, r, s$ are the mass, damping, and stiffness of BM per unit area, with $m$ a constant, $s$ a known function of $x$, and $r$ a function(al) of $x, u$.

Choosing a functional form for $r$ is a key step in model design. Many choices in the literature assume that $r$ is a local nonlinear function, $[11,7,29,13,2]$. The nonlinearity is necessary to model two tone nonlinear interaction (e.g. two tone suppression effects). However, as pointed out by Kim [15], the local form of $r$ is not sufficient to account for two tone suppression when suppressors are below the excitors in frequency (low side), unless a "second filter" is incorporated (see e.g [11]). To achieve the second filter effect on more physical grounds, micromechanical approaches have been developed to derive damping $r$ from motion of outer hair cells and other physiologically relevant parts of the cochlea, (see [15, 29, 2, 19] among others). Though this is a reasonable approach, one faces the daunting complexity of the cochlear micromechanics (see [19] for recent progress along this line). The alternative approach, first proposed in this context by Jau and Geisler [12], is phenomenological in nature. It hypothesizes that there is longitudinal coupling in the cochlear partition itself, and damping $r$ is a convolution integral of $u$ with an exponential weight function on BM. A similar idea appeared also in Lyon [21] in a coupled automatic gain control framework. Possible physiological mechanisms underlying the spatially coupled nonlocal damping were identified in Deng [3], and the resulting model was then used to process acoustic signals.

We shall adopt the latter nonlocal damping approach for choosing the function $r$. The BM damping coefficient is specified as in Jau and Geisler [12] and Deng [3]:

$$
r(x, t, u)=r_{0}(x)+\gamma \int_{0}^{L}\left|u\left(x^{\prime}, t\right)\right| \exp \left\{-\left|x-x^{\prime}\right| / \lambda\right\} d x^{\prime}
$$

where $r_{0}(x)$ is the passive BM damping; $\gamma$ and $\lambda$ are positive constants. The size of $r$ controls how sharp the BM traveling wave envelope would be. We shall take $\epsilon_{s}$ as a function of $x$ so that $\epsilon_{s}=\epsilon_{s}(x) \geq 0$ is a smooth function and is supported near the vicinity of $x=L$, for instance:

$$
\epsilon_{s}(x)=\frac{\epsilon}{1+\exp \left\{\beta\left(x_{s}-x\right)\right\}},
$$

where $x_{s}$ is a constant slightly smaller than $L ; \epsilon>1, \beta>1$, are two constants, with $\beta$ suitably large. Taking $\epsilon_{s}$ as a spatially dependent function can be considered as a way to selectively introduce damping so that possible low frequency waves near $x=L$ are damped out and there is minimal wave accumulation (or reflection) close to the helicotrema (at $x=L$ ). This turns out to be an essential stabilizing mechanism for our time domain numerical computation. We will come back to this point later. 
The coefficients $m, n$, and $s(x)$ are standard. The function $s(x)$ is based on the data of Liberman [17]:

$$
s(x)=4 \pi^{2} m(0.456 \exp (4.83(1-x / 3.5))-0.45)^{2} .
$$

The physical boundary and initial conditions are:

$$
\begin{aligned}
& p_{x}(0, t)=T_{M} p_{T}(t), \quad p(L, t)=0, \\
& u(x, 0)=u_{t}(x, 0)=0
\end{aligned}
$$

where $p_{T}(t)$ is the input sound pressure at the eardrum; and $T_{M}$ is a bounded linear operator on the space of bounded continuous functions, with output depending on the frequency content of $p_{T}(t)$. If $p_{T}=\sum_{j=1}^{J_{M}} A_{j} \exp \left\{i \omega_{j} t\right\}+$ c.c., a multitone input, c.c denoting complex conjugate, $J_{M}$ a positive integer, then $T_{M} p_{T}(t)=$ $\sum_{j=1}^{J_{M}} B_{j} \exp \left\{i \omega_{j} t\right\}+c . c$, where $B_{j}=a_{M}\left(\omega_{j}\right) A_{j}$, c.c for complex conjugate, $a_{M}(\cdot)$ a scaling function built from the filtering characteristics of the middle ear. Established data are available in Guinan and Peake [10]. The model setup is now complete.

2.2. Model Properties and Analysis We briefly go over some special regimes and solutions of the model system to illustrate the basic mathematical properties and issues. If $m=0, r=0, \epsilon=0,(2.1)$-(2.2) reduces to a second order wave equation: $n u_{t t}-(s(x) u)_{x x}=0$. When $\epsilon$ is turned on, and $\beta=0$, we have a damped second order wave equation driven by the boundary condition at $x=0$. Now suppose that all coefficients are constants; then (2.1)-(2.2) considered on the entire line admits planar wave solutions of the form:

$$
u=u_{0} \exp \{i(k x-\omega t)\}, p=p_{0} \exp \{i(k x-\omega t)\},
$$

where $k$ is the spatial wave number, $\omega$ the temporal frequency; $u_{0}$ and $p_{0}$ are respectively the amplitude of displacement and pressure. Upon substitution, it follows that:

$$
\begin{aligned}
& p_{0}=\left(-m \omega^{2}-i r \omega+s\right) u_{0} \\
& \left(k^{2} m+N\right) \omega^{2}+i\left(k^{2} r+\epsilon\right) \omega-s k^{2}=0 .
\end{aligned}
$$

If the damping coefficients $r=\epsilon=0$, then:

$$
\omega= \pm \frac{k s_{0}^{1 / 2}}{\left(k^{2} m+N\right)^{1 / 2}},
$$

showing that the system is dispersive (see [30]), i.e, $\omega=\omega(k)$ and $\omega^{\prime \prime}(k) \not \equiv 0$. Waves of different wavelength $(2 \pi / k)$ travel at different velocities. The dispersion relation (2.8) gives the group velocity:

$$
\omega^{\prime}(k)= \pm s_{0}^{1 / 2} N\left(m k^{2}+N\right)^{-3 / 2},
$$

which decays to zero as $k \rightarrow \infty$. In other words, short waves ( $k$ large) do not disperse as fast as long waves ( $k$ small) .

When damping coefficients $r, \epsilon>0(\beta=0)$, we have a damped dispersive system, and (2.8) is modified into:

$$
\omega=\frac{-i\left(k^{2} r+\epsilon\right) \pm \sqrt{4 s_{0} k^{2}\left(k^{2} m+N\right)-\left(k^{2} r+\epsilon\right)^{2}}}{2\left(k^{2} m+N\right)},
$$


with $\operatorname{Im}\{\omega\}=-\left(k^{2} r+\epsilon\right) / 2\left(k^{2} m+N\right)<0$, if the discriminant is nonnegative; otherwise:

$$
\operatorname{Im}\{\omega\}=-\frac{\left(k^{2} r+\epsilon\right) \pm \sqrt{-4 s k^{2}\left(k^{2} m+N\right)+\left(k^{2} r+\epsilon\right)^{2}}}{2\left(k^{2} m+N\right)}<0 .
$$

In either case, we see decay of planar waves. The decay rate $\operatorname{Im}\{\omega\}$ shows that if $\epsilon=0$, the decay of long waves $(|k| \ll 1)$ is very slow. Hence the decay of long waves relies on $\epsilon$. We remark that the cochlear model in $[3,6]$ also contains a socalled stiffness coupling term in equation (2.2), so the elastic response becomes: $p=$ $m u_{t t}+r(x, t, u) u_{t}+s(x) u-K(x) u_{x x}$, for some positive function $K(x)$, rendering the highest spatial derivative order four in the model. Nevertheless, it is easy to check that the additional term does not change either the dispersive nature of model solutions, or the long wave dispersion and slow decay properties.

As we will see, the long wave dispersion and slow decay properties persist when coefficients become variables, and could cause problems for numerics. However, the generic dispersive effect of the time dependent solutions is absent in the special case when the temporal dependence of solution can be factored out. This is the case when nonlinearity is absent, i.e. $r=r(x)$. Let the input $P_{T}(t)$ be a single tone, or in complex variables $P_{T}(t)=A \exp \{i \omega t\}+$ c.c. Writing solution as $u=U(x) \exp \{i \omega t\}+$ c.c., $p=P(x) \exp \{i \omega t\}+$ c.c , we derive the following boundary value problem:

$$
\begin{aligned}
& P(x)=\left(-m \omega^{2}+i r(x) \omega+s(x)\right) U, \\
& P_{x x}+\left(N \omega^{2}-\epsilon(x) i \omega\right) U=0,
\end{aligned}
$$

subject to the boundary conditions:

$$
P_{x}(0)=T_{M} A, P(L)=0 .
$$

This is the way frequency domain calculation is done, turning a time dependent problem into a steady state problem on wave amplitudes, avoiding transients and the dispersion effect. However, when nonlinearity is present, it is in general impossible to factor out the time dependence exactly, especially when the input signal consists of multiple tones. To do an accurate computation directly on system (2.1)-(2.6), one has to proceed in the time domain, or by solving the initial boundary value problem in time until transients die out and solutions approach a limiting time dependent state (almost periodic in general for multiple tones).

\section{Numerical Method}

In this section, we discuss our numerical method for solving system (2.1)-(2.6) and related numerical issues. Solutions of a cochlear model with tonal inputs behave like traveling waves during early time, and eventually settle down to time periodic states of different periods at various BM locations according to the resonance information (frequency to place mapping) encoded in the function $s(x)$. A time domain computation will provide the stabilized limiting multi-frequency oscillating states after a long enough time integration. An ideal numerical method would be one that could reach the limiting states with accuracy, low cost and speed.

Let us first briefly review some existing methods. For a cochlear model with locally nonlinear damping function $r$ (van der Pol type), a method of line discretization is implemented in [7]. Discretization in space is a Galerkin finite element method (of second order, with piecewise linear basis), and time stepping is an explicit variable 
step 4th order Runge-Kutta, found to be superior to earlier explicit time stepping schemes such as Heun's and modified Sielecki methods. A fully discrete explicit finite difference discretization, first order in time and second order in space, is carried out in $[3,6]$. The discretization can be viewed as central differencing in space and forward Euler in time. The explicit nature of these methods put a severe stability constraint on the time step, requiring long computation time to reach stable almost periodic states that we are most interested in. Also it is known that one step explicit methods such as Runge-Kutta, Heun and Euler, are prone to accumulation of truncation errors for approximating dynamic objects like limit cycles, see [9], chapter 2.

Though implicit methods typically relax the stability constraints on time step and are better for computing steady states, they are also likely to be slow due to Newton iteration at each time step, and the non-banded complicated Jacobian matrix as a result of the nonlocal damping term. When $r$ is only Lipschitz continuous in $u$, lack of smoothness is another concern for the condition of the associated Jacobian matrix. This motivates us to look into semi-implicit methods that allow larger time steps and better stability properties than explicit methods, while being cheaper and faster than fully implicit schemes.

Let us introduce our 2nd order accurate semi-implicit discretization. We denote by $u_{j}^{n}\left(p_{j}^{n}\right)$ the numerical approximation of solution $u(j h, n k)(p(j h, n k))$, where $h$ is the grid size for $x \in[0, L]$ and $k$ the time step. Let $\delta_{ \pm, h}$ be the forward/backward finite differencing operator in $x$ and $\delta_{ \pm, k}$ the forward/backward finite differencing operator in $t$. The central first differencing operator in space is $\delta_{0, h}=\frac{\delta_{+, h}+\delta_{-, h}}{2}$, and the central second differencing operator in space is $\delta_{h}^{2}=\left(\delta_{+, h}-\delta_{-, h}\right) / h$; with similar notations $\delta_{0, k}, \delta_{k}^{2}$ for the central differencing operator in time. The semi-implicit method for system (2.1-2.6) is:

$$
\begin{aligned}
& N \delta_{k}^{2} u_{j}^{n}=\frac{1}{4} \delta_{x}^{2}\left(p_{j}^{n+1}+2 p_{j}^{n}+p_{j}^{n-1}\right)-\epsilon_{j} \delta_{0, k} u_{j}^{n}, \\
& p_{j}^{n+1}=m \frac{2 u_{j}^{n+1}-5 u_{j}^{n}+4 u_{j}^{n-1}-u_{j}^{n-2}}{k^{2}}+r_{j}^{n}\left(u_{t}\right)_{j}^{n+1}+s_{j} u_{j}^{n+1},
\end{aligned}
$$

where $1 \leq j \leq J, n \geq 2, \epsilon_{j}=\epsilon_{s}\left(x_{j}\right)$, and:

$$
r_{j}^{n}=\left[r_{0, j}+\gamma h\left(\frac{1}{2}\left|u_{1}^{n+1}\right| e^{-\left|x_{j}-x_{1}\right| / \lambda}+\frac{1}{2}\left|u_{J}^{n+1}\right| e^{-\left|x_{j}-x_{J}\right| / \lambda}+\sum_{l=2}^{J-1}\left|u_{l}^{n+1}\right| e^{-\left|x_{j}-x_{l}\right| / \lambda}\right)\right],
$$

where $\left(u_{t}\right)_{j}^{n+1}$ is approximated at second order by:

$$
\begin{aligned}
\left(u_{t}\right)_{j}^{n+1} & =\left(u_{t}\right)_{j}^{n-1}+(2 k)\left(u_{t t}\right)_{j}^{n-1}+O\left(k^{2}\right) \\
& =\delta_{0, k} u_{j}^{n-1}+\frac{2 k}{N}\left(\left(p_{x x}\right)_{j}^{n-1}-\epsilon_{j}\left(u_{t}\right)_{j}^{n-1}\right)+O\left(k^{2}\right) \\
& =\delta_{0, k} u_{j}^{n-1}+\frac{2 k}{N}\left(\delta_{h}^{2} p_{j}^{n-1}-\epsilon_{j} \delta_{0, k} u_{j}^{n-1}\right)+O\left(k^{2}\right),
\end{aligned}
$$

and:

$$
\begin{aligned}
\left|u_{l}^{n+1}\right| & =\left|u_{l}^{n}+k\left(u_{t}\right)_{l}^{n}+O\left(k^{2}\right)\right| \\
& =\left|u_{l}^{n}+\left(4 u_{l}^{n-1}-u_{l}^{n-2}-3 u_{l}^{n}\right) /(-2)\right|+O\left(k^{2}\right) \\
& =\left|\left(4 u_{l}^{n-1}-u_{l}^{n-2}-5 u_{l}^{n}\right) /(-2)\right|+O\left(k^{2}\right) .
\end{aligned}
$$


In (3.4), we have used equation (2.1) once to lower the time derivative of $u$ by one order. In (3.2), (3.3) and (3.5), we have also used one sided second order differencing to approximate $u_{t t}, u_{t}$, and the trapezoidal rule to approximate the spatial integral. The variables at $t=(n+1) k$ are all linear. It follows from (3.2) that:

$$
u_{j}^{n+1}=\frac{k^{2} p_{j}^{n+1}-m\left(-5 u_{j}^{n}+4 u_{j}^{n-1}-u_{j}^{n-2}\right)+k r_{j}^{n}\left(4 u_{j}^{n}-u_{j}^{n-1}\right) / 2}{2 m+3 k r_{j}^{n} / 2+k^{2} s_{j}} .
$$

Substituting (3.6) into (3.1) to eliminate $u^{n+1}$, we get the following linear system of equations on $p^{n+1}(\lambda=k / h)$ :

$$
\begin{aligned}
& {\left[-\left(N+\frac{\epsilon_{j} k}{2}\right) k^{2} /\left(2 m+\frac{3 k r_{j}^{n}}{2}+k^{2} s_{j}\right)-\frac{\lambda^{2}}{2}\right] p_{j}^{n+1}+\frac{\lambda^{2} p_{j+1}^{n+1}}{4}+\frac{\lambda^{2} p_{j-1}^{n+1}}{4} } \\
= & N\left(-2 u_{j}^{n}+u_{j}^{n-1}\right)+\left(N+\frac{\epsilon_{j} k}{2}\right) \frac{-m\left(-5 u_{j}^{n}+4 u_{j}^{n-1}-u_{j}^{n-2}\right)+\frac{k r_{j}^{n}}{2}\left(4 u_{j}^{n}-u_{j}^{n-1}\right)}{\left(2 m+\frac{3 k r_{j}^{n}}{2}+k^{2} s_{j}\right)} \\
& -\frac{\lambda^{2}}{4}\left[2\left(p_{j+1}^{n}-2 p_{j}^{n}+p_{j-1}^{n}\right)+p_{j+1}^{n-1}-2 p_{j}^{n-1}+p_{j-1}^{n-1}\right]-\frac{\epsilon_{j} k}{2} u_{j}^{n-1}
\end{aligned}
$$

which is solved by inverting a diagonally dominant tridiagonal matrix. Once $p^{n+1}$ is computed, $u^{n+1}$ is updated from (3.6).

The first two time steps are initiated as follows. Initial condition, (2.1), and (2.2) give:

$$
p_{x x}-\frac{N}{m} p=0, \quad p_{x}(0)=f(0), p(L, 0)=0,
$$

whose solution is:

$$
p(x, 0)=\frac{f(0)}{\sqrt{N / m}\left(1+e^{-2 L \sqrt{N / m}}\right)}(\exp \{(x-2 L) \sqrt{N / m}\}-\exp \{-\sqrt{N / m} x\})
$$

At $t=k$, we found

$$
u(x, k)=k^{2} u_{t t} / 2+O\left(k^{3}\right)=\frac{k^{2}}{2 m} p(x, 0)+O\left(k^{3}\right) ;
$$

and similarly:

$$
u_{t}(x, k)=k p(x, 0) / m+k^{2} u_{t t t}(x, 0) / 2+O\left(k^{3}\right) .
$$

To find $p(x, k)$, denote $q(x)=p_{t}(x, 0)=m u_{t t t}(x, 0)+r(x, 0) u_{t t}(x, 0)$. It follows from (2.1) that $q_{x x}-N u_{t t t}(x, 0)=\epsilon(x) u_{t t}(x, 0)$, implying

$$
q_{x x}-\frac{N}{m} q=-\epsilon(x) u_{t t}(x, 0)-\frac{N}{m} r(x, 0) u_{t t}(x, 0),
$$

or a uniquely solvable two point boundary value problem on $q$ :

$$
q_{x x}-\frac{N}{m} q=-\left(N r(x, 0) / m^{2}-\epsilon(x)\right) p(x, 0),
$$

with boundary condition: $q_{x}(0)=f^{\prime}(0), q(L)=0$. 
It follows then from the definition of $q$ that:

$$
\begin{aligned}
u_{t t t}(x, 0) & =q(x) / m-r(x, 0) p(x, 0) / m^{2}, \\
u_{t}(x, k) & =k p(x, 0) / m+\frac{k^{2}}{2}\left(q(x) / m-r(x, 0) p(x, 0) / m^{2}\right) .
\end{aligned}
$$

Equations (2.1)-(2.2) at $t=k$ implies the uniquely solvable two point boundary value problem on $p(x, k)$ :

$$
p_{x x}-\frac{N}{m} p=(\epsilon(x)-N r(x, u)) u_{t}-N s(x) u / m,
$$

with boundary condition: $p_{x}(0)=f(k), p(L)=0$. Both (3.9) and (3.11) are solved with a standard second order discretization. We then will have computed $(u, p)(x, k)$. Similarly, with $k$ replaced by $2 k$, we compute $(u, p)(x, 2 k)$.

The left boundary condition $p_{x}(x, 0)=f(t)$ is discretized as: $p_{2}^{n+1}-p_{0}^{n+1}=$ $2 h f((n+1) k)$, with second order accuracy. The right boundary condition $p_{J+1}^{n+1}=0$ is exact. The semi-implicit method is now completely defined, and it reduces to an unconditionally stable implicit method for standard second order wave equation when $m=0, s$ is constant, and damping is absent ([27], chapter 8).

\section{Numerical Results and Multitone Interaction}

In this section, we present numerical results based on our model and numerical methods discussed in the last two sections. First, we list all parameters of our cochlear model and those of the associated middle ear model based on Guinan and Peake [10]. We then show computed single tones and demonstrate the dispersion effects of long waves and the role of selective longitudinal fluid damping in our model. As a test of the model tuning property, we also show the computed isodisplacement curves (at characteristic frequencies $500 \mathrm{~Hz}, 2 \mathrm{kHz}, 4 \mathrm{kHz}, 6 \mathrm{kHz}, 10 \mathrm{kHz}$ ). Subsequently, we give numerical examples of both the high side and low side suppression of two tone interaction in model solutions consistent with Figures 10.2, 10.4, 10.7 in [8]. The asymmetry of high and low side suppression is captured by the model. We then show numerical simulation of three tone interaction, presenting results on how the amplitudes of the two of the three tones change as we vary the amplitude and frequency of the third tone. Our results are qualitatively consistent with experimental data of Deng and Geisler [4] on responses of auditory neural fibers to input of three tones.

4.1. Model Parameters The parameters for $(2.2)$ are: cochlear length $3.5 \mathrm{~cm}$; mass density $0.05 \mathrm{~g} / \mathrm{cm} ; \gamma=0.2 ; \lambda=0.2 \mathrm{~cm} ; N=16.67\left(\right.$ dyne $\left./ \mathrm{cm}^{3}\right) ; r_{0}=0.001$ $\mathrm{g} /\left(\mathrm{cm}^{2} \mathrm{~ms}\right)$.

The middle ear serves as a low pass filter from sound pressure at the eardrum to the displacement of the stapes, and as a high pass filter from the sound pressure at the eardrum to the acceleration. We fit the data in [10] with the following gain factor $a_{M}(\omega)$ for each input $e^{i \omega t}$ :

$$
a_{M}(\omega)=30\left(\frac{1}{30}+0.0605 \omega^{2}\left(\left(1-\frac{\omega^{2}}{\omega_{m}^{2}}\right)^{2}+\left(2 \xi_{m} \omega / \omega_{m}\right)^{2}\right)^{-1 / 2}\right)
$$

where $\omega_{m}=4 \mathrm{kHz}$, the middle ear characteristic frequency, and $\xi_{m}=0.7$, the middle ear damping ratio. 

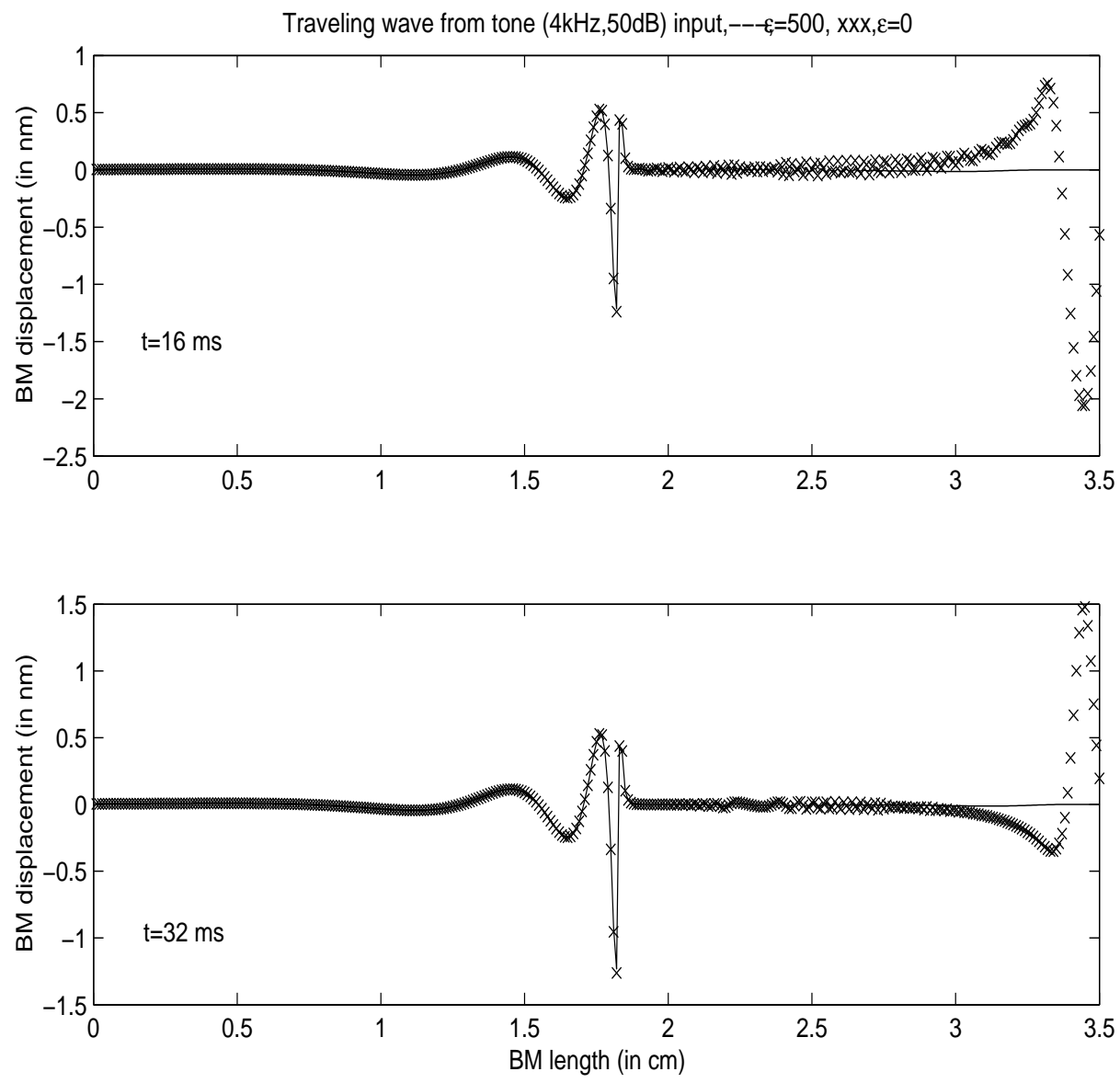

FIG. 4.1. BM displacement at $t=16 \mathrm{~ms}$ (top) and $t=32 \mathrm{~ms}$ (bottom), from a (4kHz,50dB) input, with (line) and without (cross) selective damping.

\subsection{Dispersion Effect}

We illustrate the dispersion property of time dependent solutions numerically in the case of one tone input. In the top plot of Fig. 4.1, we show the profiles of the computed traveling wave at $t=16 \mathrm{~ms}$ with and without the selective damping term, $\epsilon(x) u_{t}$. For the run, $h=0.01, k=0.01$, and $x_{s}=3, \beta=4$. We see that for a tone input of $4 \mathrm{kHz}, 50 \mathrm{~dB}$, without selective damping, the BM displacement at $t=16 \mathrm{~ms}$ contains a long wave which manages to pass through the characteristic location in the interval $(1.5,2)$ and persists near the exit $x=L$. This is due to the dispersive long waves with slow decay as we discussed in the last section. In contrast, when the selective damping term is present, the long wave is damped as it moves close to the right end point $x=L$. As the selective damping term is only effective near $x=L$, the solutions in the interior of the domain are essentially the same. In the bottom plot of Fig. 4.1, we show the BM displacement at $t=32 \mathrm{~ms}$, and the dispersive long wave persists. In other runs (not shown here), we also observed growth in the amplitude of long waves near $x=L$, with time at a linear rate. The appearance of long waves seems to be independent of numerical methods; rather, their existence is intrinsic to 


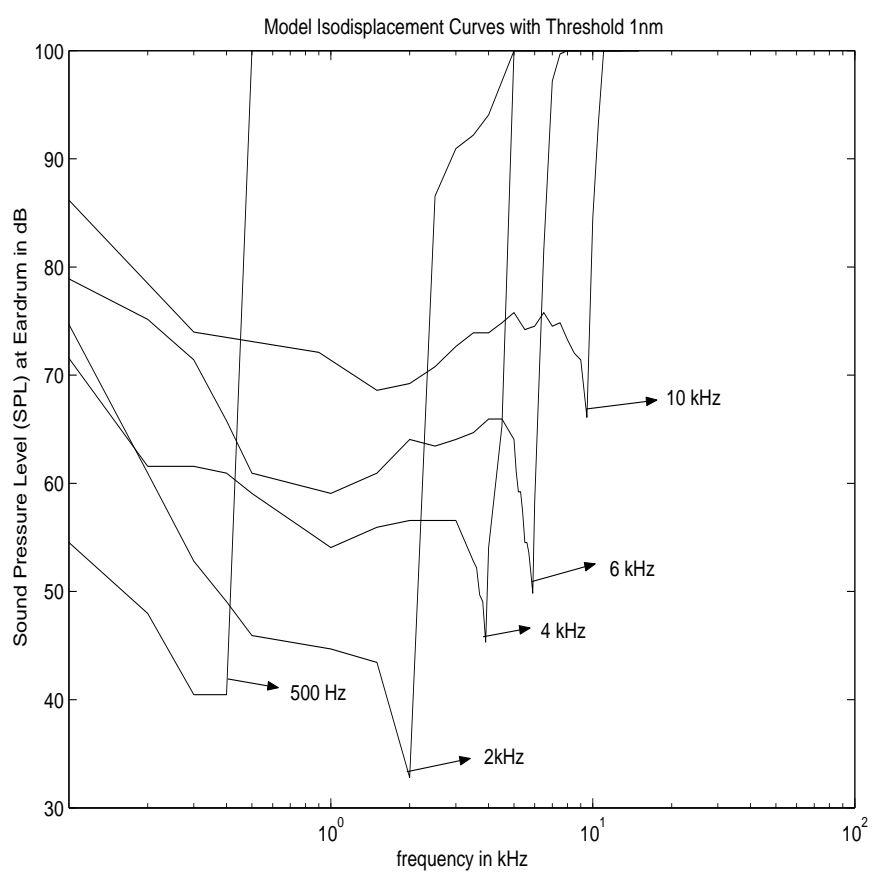

FIG. 4.2. Isodisplacement curves ( $1 \mathrm{~nm} \mathrm{BM}$ displacement) at five characteristic frequencies 500 $\mathrm{Hz}, 2 \mathrm{kHz}, 4 \mathrm{kHz}, 6 \mathrm{kHz}, 10 \mathrm{kHz}$.

the model which is dispersive.

4.3. Numerical Parameters For all our runs reported below, $h=k=0.01$, $\epsilon=500, \beta=4$, and $x_{s}=3$. The $x_{s}$ and $\beta$ values should be properly increased $\left(x_{s}\right.$ closer to $x=3.5$ ) if there are low frequencies below $300 \mathrm{~Hz}$ in the input frequencies. Smaller $h$ and $k$ values have been used to test that numerical solutions do not vary much with further refinement. The time step $k$ should be made suitably smaller if input frequencies are as large as $16 \mathrm{kHz}$ for more resolution.

4.4. Isodisplacement Curves For each input pressure wave of frequency $\omega$, there is a unique location $x_{\omega} \in[0, L]$ where the maximum BM displacement (peak in absolute value) is located. The $x_{\omega}$ is called characteristic place. Conversely, for each location $x \in[0, L]$ there is a frequency, called characteristic frequency $(\mathrm{CF})$ and denoted by $C F(x)$, such that the BM response amplitude attains the maximum at $x$. In Fig. 4.1, we see that $x_{\omega} \in(1.5,2)$ for $\omega=4 \mathrm{kHz}$. The higher the amplitude of the input wave (denoted by $A_{i n}$ ), the higher the $\mathrm{BM}$ response peak. If the level of $\mathrm{BM}$ response is set at a fixed level, such as $1 \mathrm{~nm}$, and a BM location $x$ is given, we look for an input value $A_{i n}$ so that $|u|(x)=1 \mathrm{~nm}$. Here $|u|$ is the steady state BM response for input wave $A_{i n} e^{i \omega t}+$ c.c. The plot of $A_{i n}$ as a function of $x$ (or log-frequency) gives the so called isodisplacement curve. The profile $|u|(x)$ is asymmetric about the peak, and decays rapidly to zero beyond $x_{\omega}$. Thus, it takes higher $A_{\text {in }}$ to stimulate a point $x>x_{\omega}$ to $1 \mathrm{~nm}$ than to the left. It takes least $A_{\text {in }}$ to stimulate $|u|\left(x_{\omega}\right)$ to $1 \mathrm{~nm}$. So the isodisplacement curve has a minimum at $x_{\omega}$, rises sharply at $x>x_{\omega}$ and gradually at $x<x_{\omega}$. In Fig. 4.2, we show model isodisplacement curves for five characteristic frequencies $500 \mathrm{~Hz}, 2 \mathrm{kHz}, 4 \mathrm{kHz}, 6 \mathrm{kHz}, 10 \mathrm{kHz}$. The threshold displacement is 1 

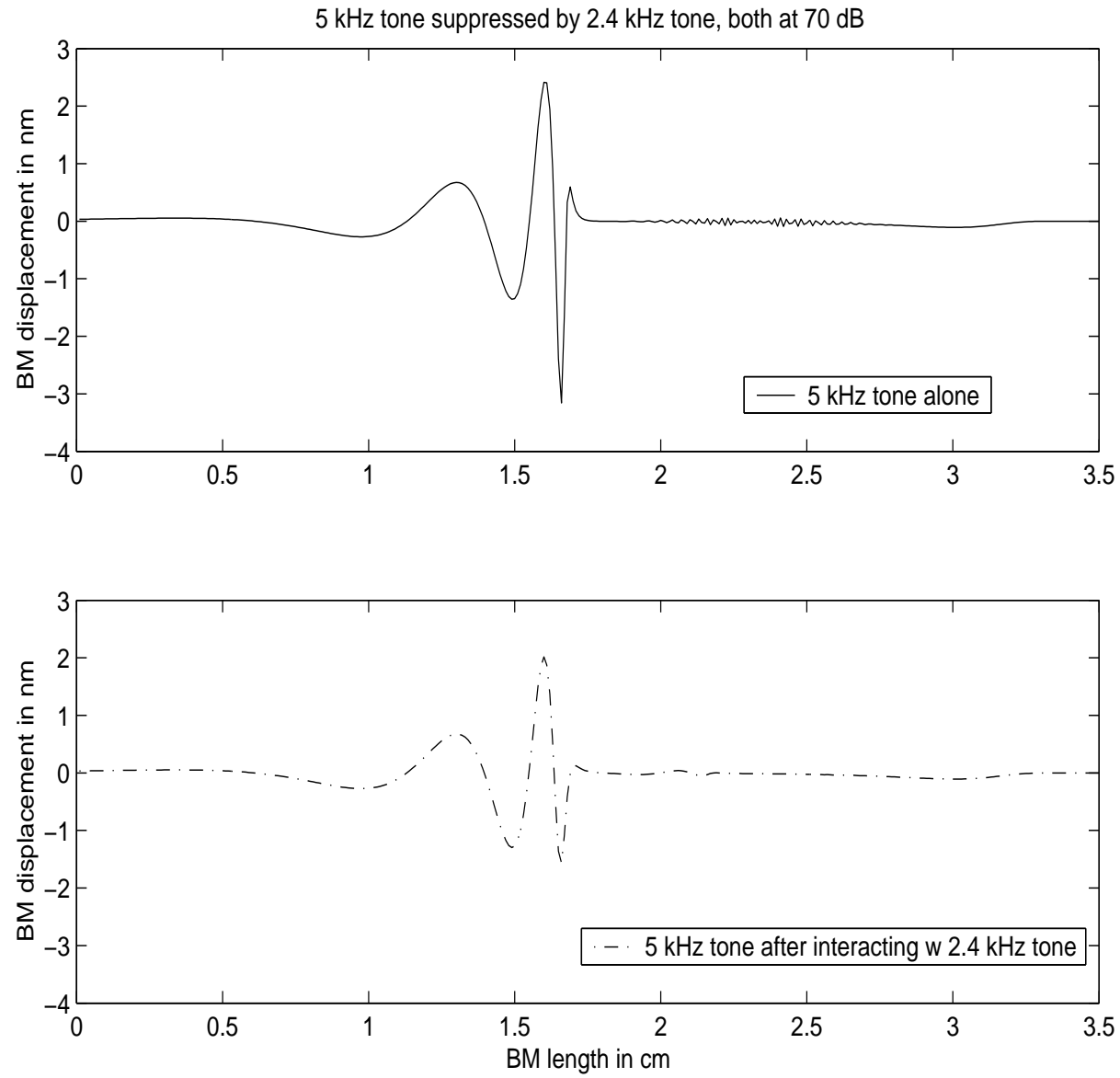

FIG. 4.3. Low side masking effect of $2.4 \mathrm{kHz}$ tone on $5 \mathrm{kHz}$ tone both at $70 \mathrm{~dB}$.

nm. The curves showed a trend of sharper tips in high frequencies, wider tips for lower frequencies, and asymmetry about the tips. The plot is comparable to the one in [23]. Isodisplacement curves are related to frequency selectivity of hearing.

4.5. Two and Three Tone Interactions Two tone interaction is welldocumented in the literature. Here we illustrate that our model gives qualitatively the same results as shown in related figures in [8]. We shall use BM displacement to detect the tonal interaction. Fig. 4.3 shows that the amplitude of a $5 \mathrm{kHz} 70 \mathrm{~dB}$ tone drops (by nearly 50\%) after the second tone of $2.4 \mathrm{kHz}$ and $70 \mathrm{~dB}$ is introduced. This reveals the so-called low side suppression.

In Fig. 4.4, we see that the $5 \mathrm{kHz}$ tone at $50 \mathrm{~dB}$ (solid line) is suppressed (by about $30 \%$ ) after interacting with $6.7 \mathrm{kHz}$ tone at $80 \mathrm{~dB}$ (plus-line), the so called high side suppression. The high side suppressor tone at $6.7 \mathrm{kHz}$ has to be much higher in amplitude than a low side suppressor tone whose frequency is at least as far away from that of the suppressed tone. This shows the asymmetry of low and high side suppression (as in [8] among others). In [5], Fig. 7a showed cat auditory neural rate response at a fixed characteristic frequency $(\mathrm{CF})$ demonstrating a suppression effect 

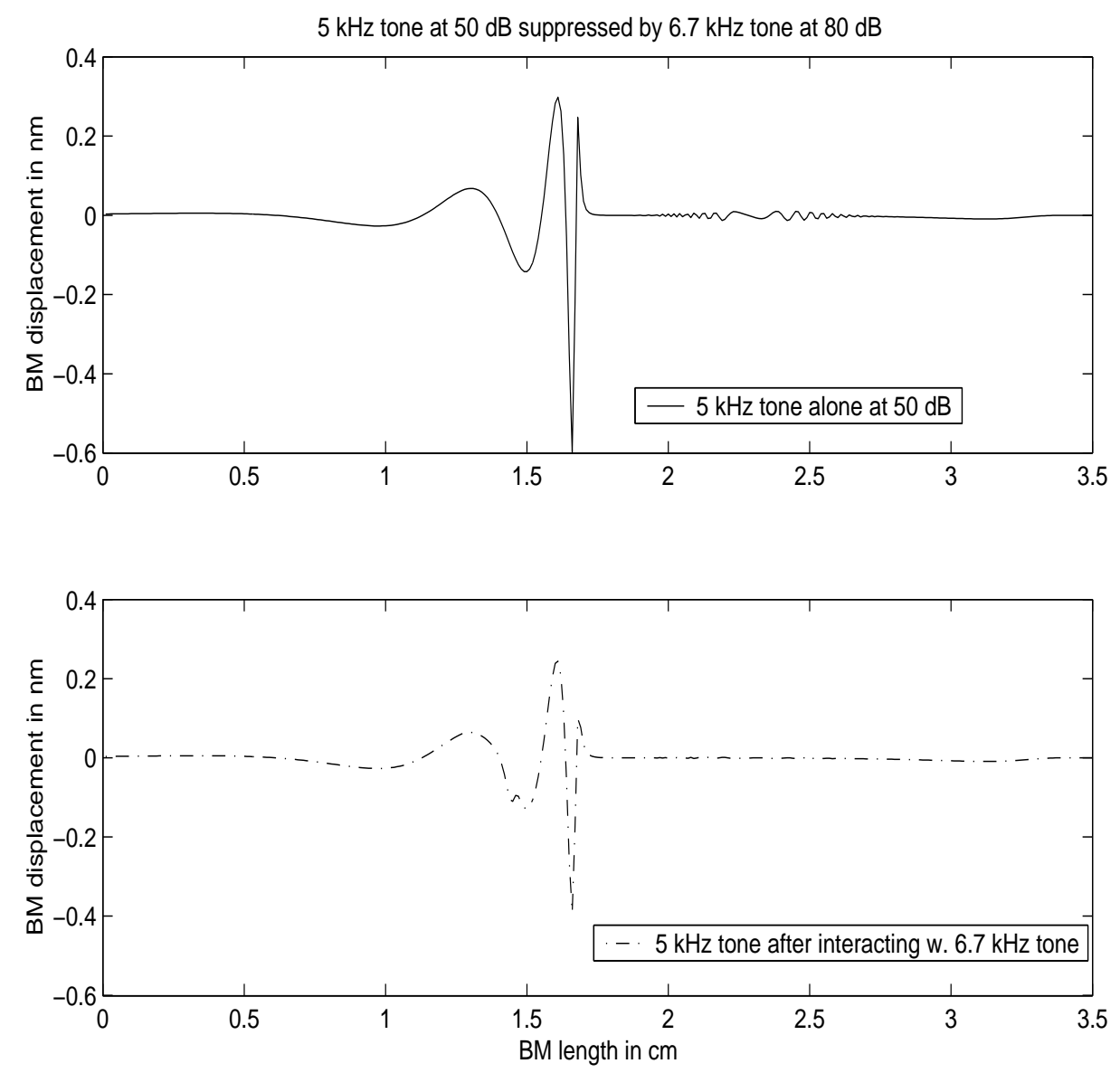

FiG. 4.4. High side masking effect of $6.7 \mathrm{kHz}$ tone at $80 \mathrm{~dB}$ on $5 \mathrm{kHz}$ tone at $50 \mathrm{~dB}$.

for two tone $\left(F_{1}, F_{2}\right)$ input, where $F_{1}$ at fixed intensity takes three frequency values from below $\mathrm{CF}$ to above $\mathrm{CF}$, for various values of the frequency $F_{2}$ at $70 \mathrm{~dB}$. In Fig. 4.5 , we showed a qualitatively similar plot computed with our model, where $\mathrm{CF}=1$ $\mathrm{kHz}, 50 \mathrm{~dB} F_{1}$ takes three frequency values $(0.9,1,1.2) \mathrm{kHz}, 70 \mathrm{~dB} F_{2}$ increases its frequencies as $1.3 \mathrm{kHz}, 1.4 \mathrm{kHz}, 1.5 \mathrm{kHz}, 1.6 \mathrm{kHz}, 1.7 \mathrm{kHz}, 1.8 \mathrm{kHz}$. The three tone interaction is much less documented in the literature. Here we shall compare our model results qualitatively with the experimental data on auditory neural response to multi-tones in [4]. Two tones are fixed at $4 \mathrm{kHz}, 4.4 \mathrm{kHz}$, both at $50 \mathrm{~dB}$. The third tone varies in frequency and in amplitude. In Fig. 4.6, we plot the BM response of two tones ( $4 \mathrm{kHz}$, plus line; $4.4 \mathrm{kHz}$, dotted line) as the third tone (line) increases its amplitude from $33 \mathrm{~dB}$ to $73 \mathrm{~dB}$ for three values of frequencies, $3.6 \mathrm{kHz}, 3.8 \mathrm{kHz}$, $4.2 \mathrm{kHz}$. We see that the two tone response decreases for increasing amplitude of the third tone, and that the suppression effect on the $4 \mathrm{kHz}$ tone is larger at $3.8 \mathrm{kHz}$ (low side) than at $4.2 \mathrm{kHz}$ (high side) for the same amplitude of the third tone. The effect of the third tone at $3.6 \mathrm{kHz}$ is even larger than that at $4.2 \mathrm{kHz}$. In Fig. 4.7, we plot similarly the response of two tones as the third tone takes on frequencies $4.3 \mathrm{kHz}, 4.6$ $\mathrm{kHz}, 4.8 \mathrm{kHz}$. The masking effect of the third tone remains, except that the second 


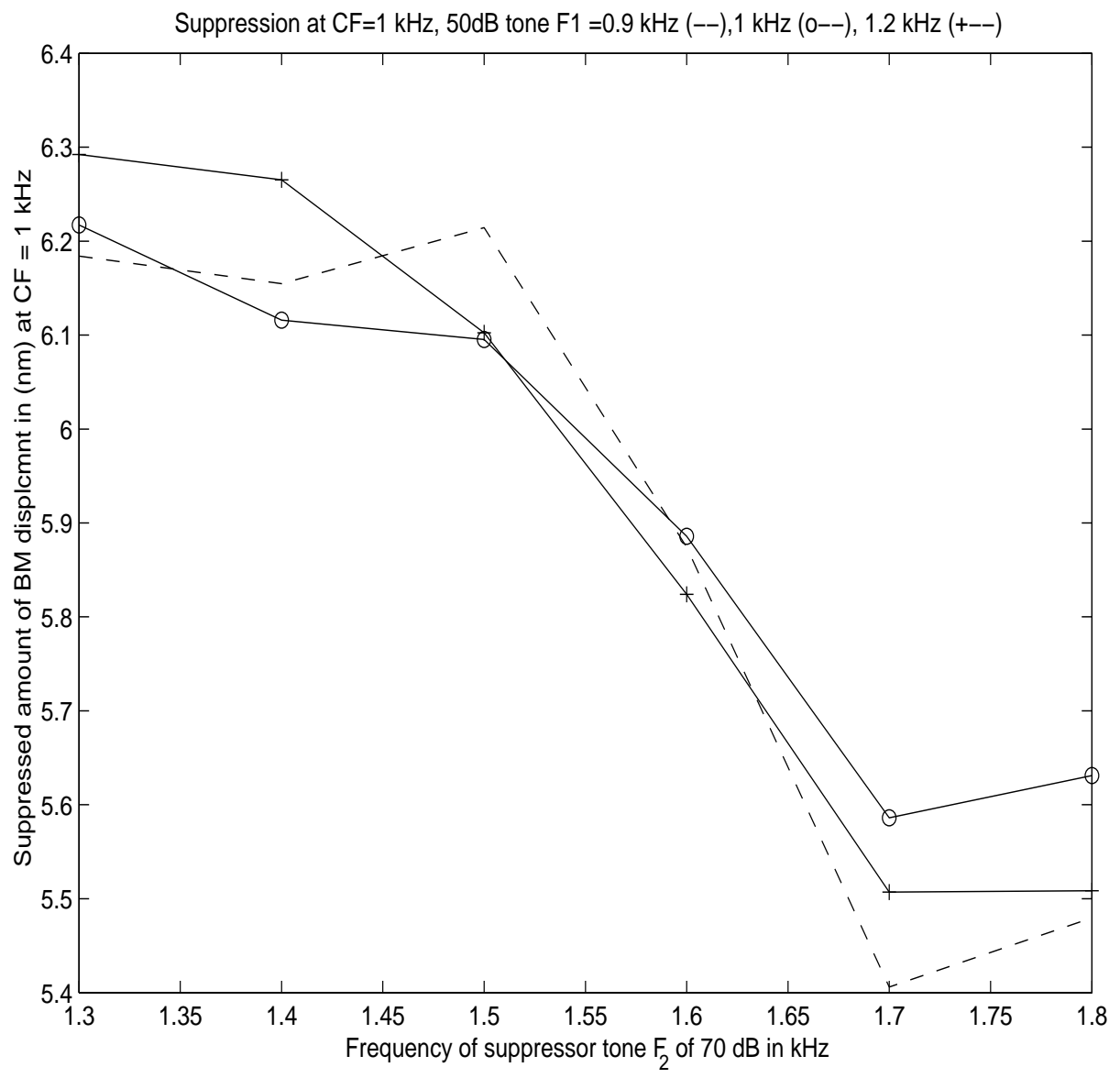

FIG. 4.5. The suppressed BM displacement (in $\mathrm{nm}$ ) at characteristic frequency $C F=1 \mathrm{kHz}$ in the presence of two tone $\left(F_{1}, F_{2}\right)$ input, for $50 \mathrm{~dB} F_{1}=0.9 \mathrm{kHz}$ (dashed line), $1 \mathrm{kHz}$ (circle-line), $1.2 \mathrm{kHz}$ (plus-line), as a function of $F_{2}$ which takes frequency values $(1.3,1.4,1.5,1.6,1.7,1.8)$ kHz at $70 \mathrm{~dB}$.

tone at $4.4 \mathrm{kHz}$ shows more difference as we observe the crossing of two tone curves when the frequency of the third tone goes through that of the second tone $(4.4 \mathrm{kHz})$. The third tone is more efficient in masking the second tone from the low side (4.3 $\mathrm{kHz})$ than from the high side $(4.6 \mathrm{kHz})$. The suppressing effect on the second tone is even weaker at $4.8 \mathrm{kHz}$. These features agree with those of Fig. 3 of Deng and Geisler [4] (e.g. compare frame 4 and frame 6 there). Strictly speaking, the neural data in [4] are synchrony masking responses, and the BM responses we computed correspond to rate masking responses. However, their data are relevant for qualitative comparison, as there is a close (positive) correlation between the rate and phase behavior (see [5]). 

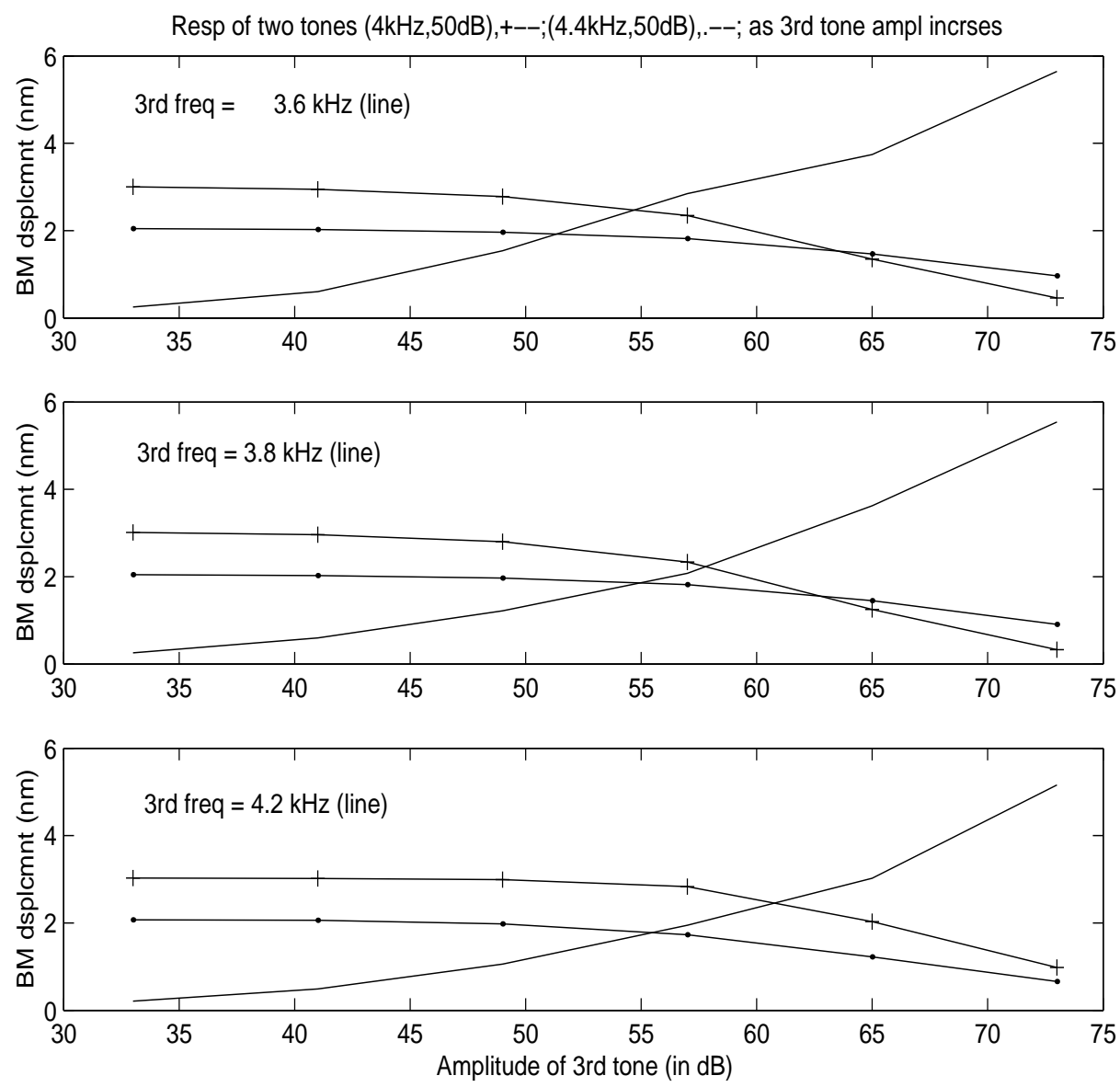

FIG. 4.6. BM response of two tones ( $4 \mathrm{kHz},+-; 4.4 \mathrm{kHz}$, dot-) as the third tone (-) increases its amplitude from $33 \mathrm{~dB}$ to $73 \mathrm{~dB}$ at three values of frequency, $3.6 \mathrm{kHz}, 3.8 \mathrm{kHz}, 4.2 \mathrm{kHz}$. 

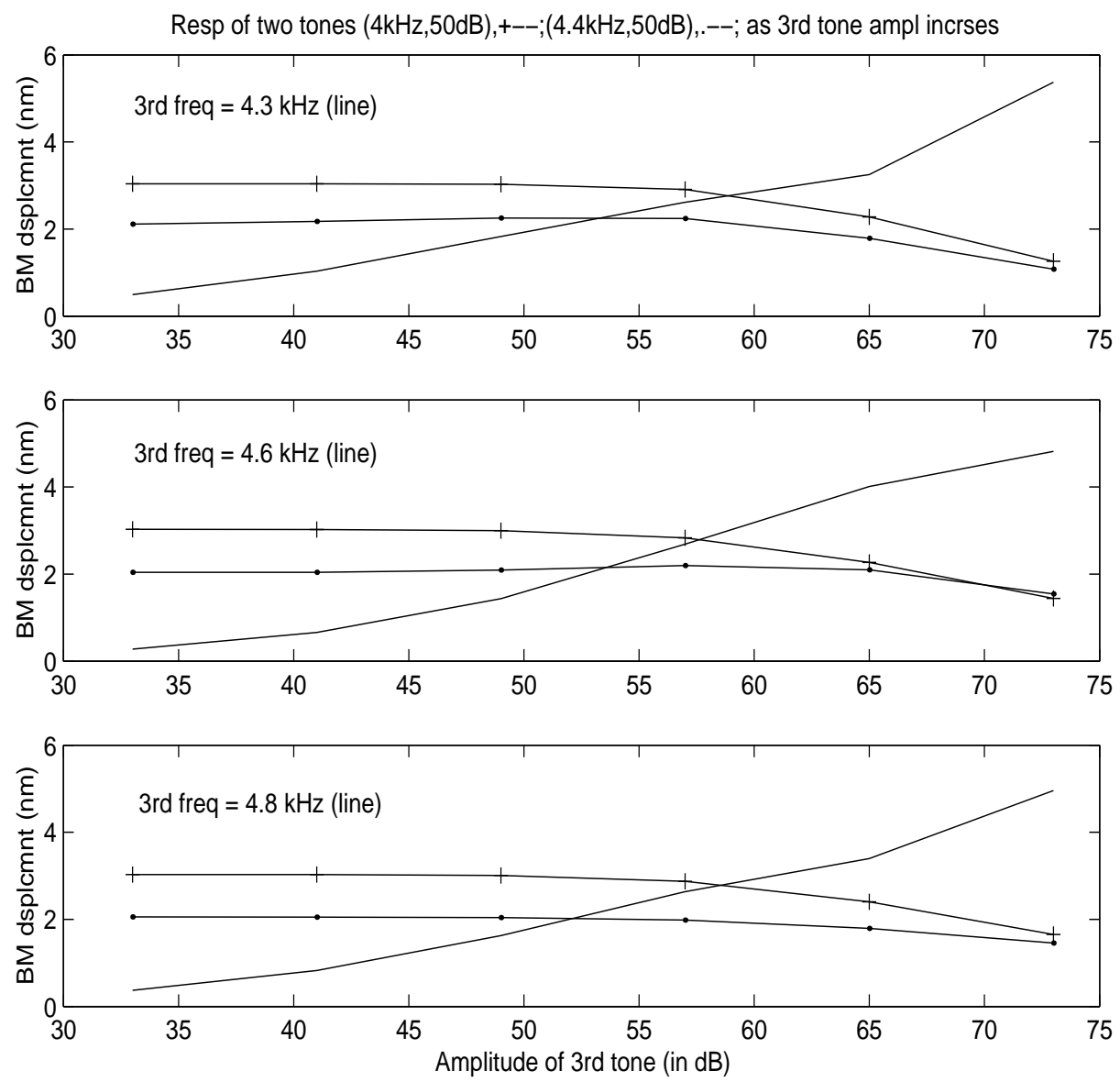

FIG. 4.7. BM response of two tones $(4 \mathrm{kHz},+-; 4.4 \mathrm{kHz}$, dot-) as the third tone (-) increases its amplitude from $33 \mathrm{~dB}$ to $73 \mathrm{~dB}$ at three values of frequency, $4.3 \mathrm{kHz}, 4.6 \mathrm{kHz}, 4.8 \mathrm{kHz}$. 


\section{Conclusions}

We discussed the dispersive property of the cochlear models for time domain computation, and slow decay of long waves. A selective longitudinal damping term has been introduced in the nonlinear nonlocal model studied previously [12,3]. The new model is computed using a semi-implicit second order finite difference method in the time domain. We presented numerical results on isodisplacement curves, two tone suppression, and three tone interaction in qualitative agreement with earlier findings of Geisler [8] and experimental auditory neural data (Deng and Geisler [4]). These encouraging results prompt further study of the masking mechanism in more complex tones and speech input based on our cochlear model.

Acknowledgments. J.X would like to thank Prof. C. D. Geisler for helpful email communications on two tone suppression and cochlear modeling. He wishes to thank Prof. J. Keller for his critical reading of the first manuscript and his constructive comments, and Prof. S. Osher for his encouragement. The authors wish to thank the Institute for Mathematics and its Applications (IMA) at the University of Minnesota for hosting the workshop on speech processing in Sept of 2000 and for providing an interactive scientific environment, where the initial collaboration began. The authors also thank Mr. M. D. LaMar for his interest and careful reading of the paper.

\section{REFERENCES}

[1] J.B. Allen, Cochlear Modeling-1980. in Lecture Notes in Biomathematics, Springer-Verlag, eds. M. Holmes and L. Rubenfeld, 43:1-8, 1980.

[2] E. de Boer Mechanics of the Cochlear: Modeling Efforts. in Springer Handbook of Auditory Research, Springer-Verlag, eds. P. Dollas and A. Popper and R. Fay, 8:258-317, 1996.

[3] L. Deng, Processing of acoustic signals in a cochlear model incorporating laterally coupled suppresive elements. Neural Networks, 5(1):19-34, 1992.

[4] L. Deng and C.D. Geisler, Responses of auditory-nerve fibers to multiple-tone complexes. J. Acoust. Soc. Amer., 82(6):1989-2000, 1987.

[5] L. Deng and C.D. Geisler, Changes in the phase of excitor-tone responses in cat auditory-nerve fibers by suppressor tones and fatique. J. Acoust. Soc. Amer, 78(5):1633-1643, 1985.

[6] L. Deng and I. Kheirallah, Numerical property and efficient solution of a transmission-line model for basilar membrane wave motions. Signal Processing, 33:269-285, 1993.

[7] R. Diependaal, H. Duifhuis, H.W. Hoogstraten and M.A. Viergever, Numerical methods for solving one-dimensional cochlear models in the time domain. J. Acoust. Soc. Amer, 82(5):1655-1666, 1987.

[8] C.D. Geisler, From Sound to Synapse. Oxford University Press, Oxford, 1998.

[9] G. Golub and J. Ortega, Scientific Computing and Differential Equations. Academic Press, San Diego, CA, 1992.

[10] J.J. Guinan and W.T. Peake, Middle-ear characteristics of anesthesized cats. J. Acoust. Soc. Amer, 41(5):1237-1261, 1967.

[11] J.L. Hall, Two-tone suppression in a nonlinear model of the basilar membrane. J. Acoust. Soc. Amer, 61:802-810, 1977.

[12] Y. Jau and C.D. Geisler, Results from a cochlear model utilizing longitudinal coupling. in Mechanics of Hearing, Martinus Nijhoff Pub., Delft Univ. Press, E. de Boer and M. Viergever, 169-176, 1983.

[13] Luc-J. Kanis and E. de Boer, Two-tone suppression in a locally active nonlinear model of the cochlear. J. Acoust. Soc. Amer, 96(4):2156-2165, 1994.

[14] J.B. Keller and J.C. Neu, Asymptotic analysis of a viscous cochlear model. J. Acoust. Soc. America, 77(6):2107-2110, 1985.

[15] D.O. Kim, An Overview Of Nonlinear And Active Models. in Lecture Notes in Biomathematics: Peripheral Auditory Mechanisms, Springer-Verlag, eds J. Allen and J.L. Hall and A. Hubbard and S.T. Neely and A. Tubis, 64:239-249, 1986.

[16] R. Leveque, Ch. Peskin, and P. Lax, Solution of a two-dimensional cochlear model with fluid viscosity. SIAM J. Applied Math, 48(1):191-213, 1988. 
[17] M.C. Liberman, The cochlear frequency map for the cat: Labeling auditory nerve fibers of known characteristic frequency. J. Acoust. Soc. Amer, 72:1441-1449, 1982.

[18] J. Lighthill, Energy flow in the cochlea. J. Fluid Mechanics, 106:149-213, 1981.

[19] K.M Lim, Physical and Mathematical Cochlear Models. PhD Dissertation, Department of Mechanical Engineering, Stanford University, 2000.

[20] B. Lutkenhoner and D. Jager, Stability of active cochlear models: need for a second tuned structure. in Lecture Notes in Biomathematics: Peripheral Auditory Mechanisms, SpringerVerlag, eds. J. Allen, J.L. Hall, A. Hubbard, S.T. Neely, and A. Tubis, 64:205-212. 1986.

[21] R. Lyon, A computational model of filtering, detection, and compression in the cochlear. IEEE International Conference on Acoustics, Speech and Signal Processing, 1282-1285, 1982.

[22] R. Meddis, L. O'Mard, and E. Lopez-Poveda, A computational algorithm for computing nonlinear auditory frequency selectivity. J. Acoust. Soc. Amer, 109(6):2852-2861, 2001.

[23] S. Neely, Mathematical modeling of cochlear mechanics. J. Acoust. Soc. Amer, 78(1):345-352, 1985.

[24] S. Osher, and L. Rudin, Feature-oriented image enhancement using shock filters. SIAM J. Numer. Analysis, 27(4):919-940, 1990.

[25] W.S. Rhode, Observations of the vibration of the basilar membrane in the squirrel monkey using the Moessbauer techniques. J. Acoust. Soc. Amer, 49:1218-1231, 1971.

[26] M. Sondhi, The Acoustical Inverse Problem for the Cochlear. Lecture Notes in Biomathematics, Springer-Verlag, eds M. Holmes and L. Rubenfeld, 43:95-104, 1980.

[27] J. Strikwerda, Finite Difference Schemes and Partial Differential Equations. Wadsworth and Brooks, Pacific Grove, CA, 1989.

[28] G. von Békésy, Experiments in Hearing. McGraw-Hill, New York, 1960.

[29] H. Wada, K. Ohyama, S. Noguchi, and T. Takasaka, Generation mechanism of toneburst-evoked otoacoustic emissions: theoretical study. in Biophysics of Hair-Cell Sensory Systems, World Scientific, Singapore, eds H.Duifhuis, J.Horst, P.van Dijk, S.M. van Netten, 94-101, 1993.

[30] G.B. Whitham, Linear and Nonlinear Waves. Wiley and Sons, New York, 1979. 